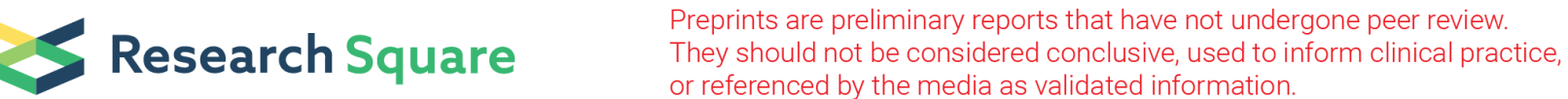

\section{Development of A Rational Framework For Understanding The Efficacy of Fecal Microbiota Transplantation In Calf Diarrhea Prevention}

Jahidul Islam

Tohoku University: Tohoku Daigaku

Masae Tanimizu

Chiba Prefectural Federation of Agricultural Mutual Aid Association

Yu Shimizu

Chiba Prefectural Federation of Agricultural Mutual Aid Association

Yoshiaki Goto

Chiba Prefectural Federation of Agricultural Mutual Aid Association

Natsuki Ohtani

Chiba Prefectural Federation of Agricultural Mutual Aid Association

Kentaro Sugiyama

Chiba Prefectural Federation of Agricultural Mutual Aid Association

Eriko Tatezaki

Chiba Prefectural Federation of Agricultural Mutual Aid Association

Masumi Sato

Chiba Prefectural Federation of Agricultural Mutual Aid Association

Eiji Makino

Chiba Prefectural Federation of Agricultural Mutual Aid Association

Toru Shimada

Chiba Prefectural Federation of Agricultural Mutual Aid Association

Chise Ueda

Chiba Prefectural Federation of Agricultural Mutual Aid Association

Ayumi Matsuo

Tohoku University: Tohoku Daigaku

Yoshihisa Suyama

Tohoku University: Tohoku Daigaku

Yoshifumi Sakai

Tohoku University: Tohoku Daigaku

Mutsumi Furukawa

Tohoku University: Tohoku Daigaku

Katsuki Usami 
Tohoku University: Tohoku Daigaku

\section{Hiroshi Yoneyama}

Tohoku University: Tohoku Daigaku

\section{Hisashi Aso}

Tohoku University: Tohoku Daigaku

\section{Hidekazu Tanaka}

Chiba Prefectural Federation of Agricultural Aid Association

\section{Tomonori Nochi ( $\nabla$ nochi@tohoku.ac.jp )}

Tohoku University https://orcid.org/0000-0003-1800-6972

\section{Research Article}

Keywords: Calf diarrhea, FMT, microbiome, metabolites

Posted Date: September 3rd, 2021

DOl: https://doi.org/10.21203/rs.3.rs-815397/v1

License: (c) (i) This work is licensed under a Creative Commons Attribution 4.0 International License. Read Full License 


\section{Abstract}

Background: Establishing fecal microbiota transplantation (FMT) to prevent multifactorial diarrhea in calves is challenging because of differences in farm management practices, the lack of optimal donors, and recipient selection. In this study, the underlying factors of successful and unsuccessful FMT trials are elucidated, and the potential markers for predicting successful FMT are identified using fetal metagenomics via $16 \mathrm{~S}$ rRNA gene sequencing, fecal metabolomics via CE-TOFMS, and machine-learning approaches.

Results: 20 FMT trials, in which feces from healthy donors were intrarectally transferred into recipient diarrheal calves, were conducted with a success rate of $70 \%$. Selenomonas was identified as a microorganism genus that showed significant donor-recipient compatibility in successful trials. A strong positive correlation between the microbiome and metabolome data, which is a prerequisite factor for FMT success, was confirmed by Procrustes analysis in successful trials $(r=0.7439, P=0.0001)$. Additionally, weighted correlation network analysis confirmed the positively or negatively correlated pairs of bacterial taxa (family Veillonellaceae) and metabolomic features (i.e., amino acids and short chain fatty acids) responsible for FMT success. Further analysis aimed at establishing criteria for donor selection identified the genus Sporobacter as a potential biomarker in successful donor selection. Low levels of metabolites, such as glycerol 3-phosphate, dihydroxyacetone phosphate, and isoamylamine, in the donor or recipients prior to FMT are predicted to facilitate FMT.

Conclusions: Overall, we provide the first substantial evidence of the factors related to FMT success or failure; these findings could improve the design of future microbial therapeutics for preventing diarrhea in calves.

\section{Background}

Fecal microbiota transplantation (FMT), in which fecal contents from healthy donors are transplanted into diseased patients with the intention of normalizing or restoring healthy gut microbiota, is considered a promising therapeutic for dysbiosis-related diseases [1, 2]. Infections caused by recurrent Clostridium difficile can be treated by FMT with a high success rate [3, 4], and FMT is used as a treatment option on patients with IBD, IBS, and autoimmune disorders [5]. Recently, the efficacy of FMT for the treatment of multifactorial calf diarrhea (CD) has also been confirmed [6]. However, maximizing the effects of FMT as a treatment for $\mathrm{CD}$ remains a challenge because the donor or recipient may be selected inappropriately due to gut microbiota compositions varying even within healthy populations as a result of environmental factors, farm management, and calf age [3]. These obstacles may increase the lack of reproducibility or the chance of FMT failure when attempting to prevent CD.

$\mathrm{CD}$ is a common enteric disease that causes enormous financial losses in the livestock industry worldwide due to high morbidity and mortality [7]. Infectious CD is mainly caused by infection from viruses (e.g., rotavirus and coronavirus), bacteria (e.g., enterotoxigenic Escherichia coli, Salmonella, and 
Clostridium perfringens), protozoa (e.g., Cryptosporidium parvum and coccidia), or a combination of such pathogens $[8,9]$. However, these enteropathogens are often found in healthy calves, indicating that their presence is not always causative [10]. Therefore, clinical veterinarians face a huge challenge to provide correct $\mathrm{CD}$ diagnosis; indeed, misdiagnosis may promote the improper use of antibiotics and the emergence of antibiotic-resistant bacteria [11, 12].

Microbial symbiosis in the gastrointestinal tract is vital for host regulation of mucosal immunity and nutrients, and for providing resistance against pathogen colonization [13]. Microbial colonization of newborns is pivotal to healthy development. Following FMT, the beneficial microbiota of healthy donors can restore the microbial community of recipient calves suffering from CD. To ensure the efficacy and durability of FMT as a disease cure, both the microbial consortium from the donor and the existing endogenous microbiota in the recipients must function collaboratively [14]. Therefore, although FMT may be an alternative therapeutic for CD prevention, the following must still be investigated and understood: 1) the beneficial microorganisms present in the feces of FMT donors, 2) the functional microorganisms and metabolites that lead to success (or failure) of FMT, and 3) the characteristics of FMT recipients that facilitate the engraftment and maintenance of donor-derived beneficial microorganisms and metabolites.

To establish FMT as a common therapeutic with high repeatability and reproducibility in veterinary medicine, the logistics of selecting donors and optimizing efficacy must be developed further. Typical approaches to selecting donors in experimental FMT trials for CD prevention involve using a single healthy donor or randomly selecting multiple donors from a set of screened potential donors [15], which is often not practical. Therefore, we aimed to address this issue by introducing the random-forest (RF) classification method. The RF classification is an effective machine-learning approach that can be used to elucidate the relationship between high-dimensional microbiota data and disease attributes. With this model, structural changes in existing microbiota data are analyzed to make new predictions and evaluate the subset of microbial taxa with relative abundances that are positively or negatively correlated with disease or therapeutic-related target variables $[16,17]$. Therefore, in the present study, microbial composition data from FMT trials along with additional field data obtained from a sufficient number of calves with or without diarrhea were subjected to RF classification to determine the optimal donor prediction for successful FMT in future.

\section{Methods}

Animals. In total, 198 healthy calves and calves with diarrhea, bred at 20 different farms at Chiba prefecture in Japan, were used in this study. Among these animals, healthy calves $(n=20)$ and calves suffering from intractable diarrhea $(n=20)$ were randomly selected as donors and recipients for FMT, respectively. The severity of diarrhea in recipients was graded according to fecal consistency. Fecal scores were assigned as follows: $1=$ normal (retains form), 2 = soft (flows across a surface), $3=$ muddy (liquid), and 4 = severe diarrhea (very watery). Fecal samples collected from FMT donors and recipients were subjected to etiologic evaluation against 10 major enteropathogens that often cause CD (i.e., bovine leukemia virus, bovine viral diarrhea, rotavirus, C. perfringens, C. parvum, coccidia, Salmonella spp., 
coronavirus, pathogenic E. coli, and nematodes). To estimate the diarrheal condition as well as colonic motility, the water content in feces was investigated by comparing the weight before and after freezedrying and using the following calculation: water content $(\%)=100 \times$ (wet weight - dry weight) / wet weight according to a previous study [18].

FMT Procedure. Fresh feces ( 100 g) were collected from healthy donors and suspended in 200-250 ml of sterilized saline. Fecal suspensions were filtered using medical gauze to remove large particles and immediately transferred intrarectally into recipients suffering from intractable diarrhea. The efficacy of FMT trials was monitored based on physical condition, diarrheal status, and clinical status. Fecal samples were collected from donors on the day of FMT and from recipients before FMT (day 0), 1 day after FMT, and 7 days after FMT. They were then stored at $-80^{\circ} \mathrm{C}$ for metabolomics and ELISA analyses or in Stool Nucleic Acid Collection and Preservation Tubes (Norgen) at room temperature for metagenomics analysis. Blood samples were also collected from the jugular veins of recipients at day 0 and 7 for plasma analyses.

Amplification of 16S rRNA gene by PCR. Genomic DNA was extracted from feces using a Stool DNA Isolation Kit according to the manufacturer's protocol. This DNA was subjected to PCR according to previous methods [19]. The V3 and V4 regions of bacterial 16S rRNA were amplified from genomic DNA using PCR with PrimeSTAR HS DNA Polymerase (TAKARA) and the following primers: forward primers mixed (5'-TGCTCTTCCGATCTGACNNNCCTACGGGNGGCWGCAG-3', 5'-

TGCTCTTCCGATCTGACNNNNCCTACGGGNGGCWGCAG-3', 5'-

TGCTCTTCCGATCTGACNNNNNCCTACGGGNGGCWGCAG-3', and 5'-

TGCTCTTCCGATCTGACNNNNNNCCTACGGGNGGCWGCAG-3'); reverse primers mixed (5'-

CGCTCTTCCGATCTCTGNNNGACTACHVGGGTATCTAATCC-3', 5'-

CGCTCTTCCGATCTCTGNNNNGACTACHVGGGTATCTAATCC-3', 5'-

CGCTCTTCCGATCTCTGNNNNNGACTACHVGGGTATCTAATCC-3', and 5'-

CGCTCTTCCGATCTCTGNNNNNNGACTACHVGGGTATCTAATCC-3'). The adaptor tag sequence is singly underlined, whereas the spacer sequences are double underlined. The PCR fragments obtained from the first round of PCR were purified using AMPure XP and then amplified in a second round of PCR using the following primers: forward (5'-

CAAGCAGAAGACGGCATACGAGATxxxxxxxxxGTGACTGGAGTTCAGACGTGTGCTCTTCCGATCTGAC-3') and reverse $(5 '-$

AATGATACGGCGACCACCGAGATCTACACxxxxxACACTCTTTCCCTACACGACGCTCTTCCGATCTCTG-3'), which included nine and five base indices shown as "xxxxxxxxx" and "xxxxx," respectively, to distinguish each sample. All PCR products were sequenced using the MiSeq platform (Illumina) with the MiSeq Reagent Kit v2 (500 cycles).

Metagenomics and functional prediction analyses. Demultiplexed raw sequences were acquired from the BaseSpace Sequence Hub (Illumina), and sequences were analyzed using QIIME 2 (version 2020.2) [20]. The quality of joined sequences was filtered using the q2-demux plugin, which was followed by denoising with DADA2 to cluster OTUs and generate a feature table for further analysis. The data from processed 
sequencing were assigned and aligned to the Greengenes reference database at $99 \%$ sequence similarity [21]. LEfSe was applied to determine the most discriminant taxa among the groups based on the feature table [22]. For statistical analyses and visual exploration, an OTU table with taxa in plain format and metadata file were analyzed using MicrobiomeAnalyst [23]. The functional potential of microbiome data from different groups was predicted based on the 16S rRNA data using Piphillin, which was established by using the KEGG database and BioCyc reference database to assign functional properties [24].

CE-TOFMS metabolomics analysis. Metabolomics analysis using fecal samples was conducted at the Human Metabolome Technologies, Inc [25]. Specifically, the samples were dried using a freeze dryer (TAITEC) and analyzed by CE-TOFMS (Agilent). The metabolite standards, instrumentation, and CETOFMS conditions used in this study were identical to those previously described [25]. The identified metabolites were quantified by comparing their peak areas with those of authentic standards using ChemStation software (Agilent Technologies).

WGCNA analysis. To describe the correlations of microbiomes and metabolites associated with the success or failure of FMT, coexpression networks were constructed using the WGCNA (v1.70-3) package in $\mathrm{R}$ [26]. WGCNA is a bioinformatics application in which microbial taxa form modules and link to specific traits of interest. The modules were obtained using the automatic network construction function with the default settings, except that the power was 10, TOMType was signed, and minModuleSize was 10. Modules were defined as clusters of highly interconnected microbial taxa; those taxa within the same cluster had high pairwise correlation coefficients. The modules obtained from each analysis were further analyzed in MicrobiomeAnalyst 4.0 to generate heatmaps [23].

Multivariate data analysis. Multivariate statistical tools, including unsupervised PCA and supervised PLSDA, were employed to reduce and visualize the complex metabolomics datasets [27]. In PLS-DA, parameters of R2X and Q2 were used to evaluate model quality and predictive ability, respectively. Values $>0.5$ indicated that the models were robust and the predictions reliable. Score plots of the PLS-DA were applied to visualize the separation between the studied groups and loading plots were used to find candidate biomarkers responsible for the separation. These biomarkers were considered to differentiate metabolites that were selected on the basis of VIP values $>1.0$ as a threshold. P-values were corrected for multiple testing using a false-discovery rate (Q-value) method [28]. Procrustes analysis for both the microbiome and metabolome was conducted using the Procrustes function in the vegan $\mathrm{R}$ package.

Statistics. Alpha and beta diversity statistics were obtained using the QIIME 2 scripts diversity plugin [20]. In the calculation of alpha diversity metrics, normalization was performed using the "rarefaction" QIIME 2 process with standard parameters and by setting the max_rare_depth (upper limit of rarefaction depths) to the mean sample size. Alpha diversity metrics were calculated using Shannon's diversity index and phylogenetic diversity. The beta diversity of the microbial profile was calculated using the QIIME 2 "diversity beta-group-significance" script. Phylogenetic (unweighted UniFrac distance) beta diversity metrics were calculated and graphically visualized by three-dimensional PCoA representations and verified using a nonparametric PERMANOVA test with 999 permutations. Correlations between selected 
microorganisms and metabolites were assessed by Pearson's correlation test using "cortest" in R. To create the classifiers, RFs comprising 500 trees were computed using the default settings of the "randomForest" function implemented in the randomForest $\mathrm{R}$ package [29]. All other statistical analyses were performed in Graphpad Prism version 7 (GRAPHPAD Software).

\section{Results}

FMT efficacy in CD prevention. Twenty FMT trials were conducted to treat recipient calves suffering from refractory CD (Fig. 1A). FMT trial efficacy was determined by diarrheal score, physical appearance, and performance from enteropathogenic microbial studies using feces collected from recipients just before and 1 week after treatment. A representative fecal sample collected from successful FMT trials (Fig. 1B) showed that they reduced the incidence of diarrhea in recipient calves. Consistent with a previous study [6] FMT was effective as a therapeutic to cure refractory CD: 14 of 20 trials were successful in the present study (Additional file 1: Table S1). However, the results gave rise to a new challenge, i.e., identifying the essential factors responsible for not only successful but also unsuccessful FMT trials. Indeed, the clinical distinction between successful (70\%) and unsuccessful (30\%) trials was clear: diarrheal score and fecal water content decreased significantly after FMT only in successful trials (Fig. 1C and D). A classical method was employed in fecal tests to identify causative enteropathogens: $70 \%$ (14/20) of recipients were diagnosed with infectious $\mathrm{CD}$, as indicated by the presence of $C$. perfringens, $C$. parvum, rotavirus, and/or coccidia in multiple calves with diarrhea (Fig. 1E). Interestingly, C. perfringens was still detected frequently 7 days after FMT regardless of the symptomatologic recovery (Fig. 1E). In addition, 25\% (5/20) and $5 \%(1 / 20)$ of calves were diagnosed with dietary enteritis and weak calf syndrome, respectively. The cure rate for dietary enteritis was $100 \%$ and $0 \%$ in successful and unsuccessful trials, respectively (Additional file 2: Fig. S1). The efficacy of FMT was identical among different species of recipients as the ratios of successful trials in Holstein, Japanese beef cattle (Wagyu), and F1 were 83.3\% (5/6), 66.7\% $(6 / 9)$, and $60.0 \%(3 / 5)$, respectively (Fig. 1F). Importantly, the combination of donor and recipient species did not affect FMT efficacy as $88.89 \%$ (8/9) and 54.55\% (6/11) were successful when xenotransplantation and allogenic transplantation were conducted, respectively (Fig. 1G). Blood tests showed that the levels of most components did not differ between successful and unsuccessful recipients before and 7 days after FMT (Additional file 2: Fig. S2). However, the concentration of total cholesterol was higher in successful recipients than the concentration in unsuccessful recipients 7 days after (although not before) FMT (Additional file 2: Fig. S2). Additionally, higher levels of $\mathrm{Y}$-GT were found high in successful recipients than were found in unsuccessful recipients before (but not 7 days after) FMT (Additional file 2: Fig. S2). Overall, these results suggest that total cholesterol and $\mathrm{Y}$-GT may be useful as blood biomarkers to monitor FMT efficacy [30, 31].

\section{Difference in fecal microbial composition of healthy donors and diarrheal recipients before and after}

FMT: successful vs. unsuccessful trials. The microbial compositional difference between successful and unsuccessful trials was first assessed using 16S rRNA gene sequencing; high-quality sequences were clustered into operational taxonomic units (OTUs) according to a cut-off of $97 \%$ sequence similarity using the QIIME2 bioinformatics platform [20]. Analysis at the phylum level did not show a clear 
difference between successful and unsuccessful trials (Fig. 2A; Additional file 2: Fig. S3); however, analysis at the family level showed that Veillonellaceae was more abundant in successful trials, whereas Lachnospiraceae, Ruminococcaceae, Methanobacteriaceae, Peptostreptococcaceae, Odoribacteraceae, and Barnesiellaceae were observed significantly more in unsuccessful trials (Fig. 2B; Additional file 2: Fig. S4). According to genus level analysis, Clostridium and Methanobrevibacter were significantly more abundant after FMT in unsuccessful trials (Fig. 2C; Additional file 2: Fig. S5). Alpha diversity analysis [32] was conducted using the Shannon index and phylogenetic diversity (faith's PD) in QIIME2; results showed that more diverse and distinct bacterial communities was present in donors compared with the bacterial communities in recipients with diarrhea before FMT in both successful and unsuccessful trials (Fig. 2D). In the recipients from both trial cases, alpha diversity indexes tended to increase after FMT (Fig. 2D). Beta diversity analysis was conducted using nonparametric permutational multivariate ANOVA (PERMANOVA) test with 999 permutations [33] to measure the compositional similarities between bacterial communities within groups of samples. For this, abundance data based on unweighted UniFrac distance matrices were analyzed; results showed that significant divergences existed between groups (Fig. 2E). Specifically, in successful trials, statistical differences in distance were observed between donors (D-success) and recipients just before FMT (R-0-success) and between D-success and recipients 1 day after FMT (R-1success), but not between D-success and recipients 7 days after FMT (R-7-success) (Additional file 1: Table S2). In contrast, in unsuccessful trials, there were no significant differences between D-failure and R-0-failure, D-failure and R-1-failure, and D-failure and R-7-failure (Additional file 1: Table S2). Thus, in successful but not unsuccessful trials, recipient calves gained a healthy donor microbiome composition and showed signs of donor-recipient engraftment in their gastrointestinal tract at day 7 .

\section{Identification of microorganisms responsible for the success and failure of FMT in donors and recipients.}

We hypothesized that, in successful trials, FMT-induced remission of diarrhea might be the result of a commensal bacterial community being generated and CD-causative pathogens being eradicated. To contextualize our findings, linear discriminant analysis effect size (LEfSe) [22] analysis was conducted to investigate the differential abundance of microbial taxa between successful and unsuccessful trials in donors and recipients. When donors were compared by the success or failure of FMT, the family Prevotellaceae and genera Prevotella, Succinispira, and Selenomonas were found to be statistically more differentially abundant in the successful trials, whereas the genera Lactonifactor, Alistipes, Roseburia were differentially more abundant in the unsuccessful trials (Fig. 3A). Discriminatory taxa were not identified, however, in either successful or unsuccessful recipients 1 day after FMT. In successful recipients, the genus Lactobacillus showed increased differential abundance prior to FMT (R-0-success) at day 0 , whereas the family Veillonellaceae and genera Selenomonas, Acidaminococcus, and Collinsella were significantly more abundant 7 days after FMT (R-7-success) (Fig. 3B, C). In unsuccessful recipients, multiple bacteria were identified as discriminatory taxa prior to FMT (R-0-failure) and 7 days after FMT (R7 -failure). Among these discriminatory taxa, the phyla Tenericutes and Spirochaetes were also found in unsuccessful donors (D-0-failure) (Fig. 3A, B). It should be emphasized that the genus Selenomonas was also found in successful donors (D-0-success), suggesting that it may act as a signature microbe and could have the potential to ensure donor-recipient compatibility (Fig. 3A, C). Moreover, further analysis 
using Piphillin [24], an algorithm applied to interpret the potential functions of a microbial community, identified 264 Kyoto Encyclopedia of Genes and Genomes (KEGG) pathway modules as FMT-related pathways (Additional file 3: Dataset S1). Subsequent LEfSe analysis based on the results from Piphillin revealed that the pathways ko01040 (Biosynthesis of unsaturated fatty acids) and ko00521 (Streptomycin biosynthesis) were enriched in successful and unsuccessful donors, respectively (Fig. 3D). Pathways ko00520 (Amino sugar and nucleotide sugar metabolism) and ko01210 (2-0xocarboxylic acid metabolism) were significantly enriched before FMT (day 0 ) in successful and unsuccessful recipients, respectively, whereas ko00520 and ko01100 (Glycolysis, gluconeogenesis, TCA cycle) were identified as enriched metabolic pathways activated 7 days after FMT in successful recipients (R-7-success) (Fig. 3E).

\section{Difference in the fecal metabolite composition of healthy donors and diarrheal recipients before and after} FMT: successful vs. unsuccessful trials. To investigate the effects of FMT-induced changes in the gut microbiome on intestinal metabolism, most fecal samples collected from 12 FMT trials (9 successful trials; 3 unsuccessful trials) were analyzed by capillary electrophoresis coupled with time-of-flight mass spectrometry (CE-TOFMS). Results showed that 366 total peaks composed of cations (214 peaks) and anions (152 peaks), and 264 peaks including 159 cations and 105 anions, were attributable to known standard metabolites that could be quantified (Additional file 2: Fig. S6). Consistent with the microbial composition, principal component analysis (PCA) showed widely dispersed data points on plots of fecal metabolomes in successful and unsuccessful FMT trials (Fig. 4A). The distance among the groups based on PC1 scores are illustrated in Additional file 1: Table S3. Interestingly, partial least squares-discriminate analysis (PLS-DA) showed that there were metabolites compositional differences in both donor and recipients between the trials (Additional file 2: Fig. S7). Specifically, when comparing successful and unsuccessful donors on the day of FMT (D-0-success vs. D-0-failure), 65 potential metabolites with a variable importance in projection (VIP) score [34] $>1$ were identified from the PLS-DA model (Additional file 3: Dataset S2); the top 15 metabolites, including dihydroxyacetone phosphate, glucose 6-phosphate, and glycerol 3-phosphate, are shown in Fig. 4B. By comparing the successful and unsuccessful recipients, 85, 74, and 73 metabolites with VIP scores $>1$ were identified following analysis prior to FMT (R-0-success vs. R-0-failure), 1 day after FMT (R-1-success vs. R-1-failure), and 7 days after FMT (R-7success vs. R-7-failure), respectively (Additional file 3: Dataset S3-5). The top 15 metabolites are shown for each analysis in Fig. 4B. Furthermore, changes in the major metabolites of amino acid metabolism, lipid and fatty acid metabolism, and sugar metabolism were investigated (Fig. 4C; Additional file 2: Fig. S8 and S9). In addition, other compounds responsible for lipid and fatty acid metabolism were identified based on their relative area due to the lack of standards available (Fig. 4D; Additional file 2: Fig. S10). In successful but not unsuccessful recipients, amino acid metabolism was high prior to FMT (R-0-success) and 1 day after FMT (R-1-success) compared with that observed 7 days after FMT (R-7-success). Specifically, glucogenic amino acids (alanine, aspartate, glutamine, glutamic acid, methionine, proline, serine, threonine, and valine), glucogenic \& ketogenic amino acids (phenylalanine and tyrosine), and ketogenic amino acids (leucine and lysine) differed significantly among these groups (Additional file 2: Fig. S8). The polyamines spermidine and putrescine, and another diamine cadaverine were also elevated in successful trials (Additional file 2: Fig. S11). These results suggest that successful FMT may be 
accompanied by changes in metabolites and especially by decreases in concentrations of amino acids related to FMT-induced changes in gut microbiota.

\section{Microbiome data correlate with metabolite profiles in successful but not unsuccessful trials. To}

investigate microbiota-metabolite correlations in successful and unsuccessful trials, Procrustes analysis [35] and the mantel test were performed using the vegan package in $\mathrm{R}$ [36]. Procrustes analysis of Euclidean distances between metabolomes and unweighted UniFrac distances highlighted the significant association between the microbiota taxonomic and metabolic profiles. Specifically, significant relatedness (Procrustes correlation $=0.7439, P=0.0001$ ) between the microbiota and metabolites was observed in successful trials (Fig. 5A), whereas a relatively low correlation (Procrustes correlation = 0.3237, $P=0.0407$ ) was observed in unsuccessful trials (Fig. 5B). In addition, group-specific Procrustes analyses aimed at distinguishing between successful and unsuccessful FMT, donors and recipients, and results before and after FMT in recipients showed that a correlation was only observed in successful donors ( $D$-0-success, $p=0.0037$ ) and successful recipients 7 days after FMT (R-7-success, $p=0.0022$ ) (Additional file 2: Fig. S12). Furthermore, the functional correlation between alternations in the microbiota and metabolites was assessed using Pearson's correlation based on 14 potential bacterial genera (shown in Fig. 2C) and metabolites (with VIP scores > 1.8; shown in Fig. 4B) that could have contributed substantially to the differences between groups. In donors, Lactonifactor and Roseburia were positively correlated with 3-phosphoglysercic acid (a major compound in glycolysis) in successful trials, whereas Succinivibrio was negatively correlated with pimelic acid and P-aminoenzioc acid in unsuccessful trials (Fig. 5C). In recipients, prior to FMT, Clostridium and Roseburia were positively correlated with fructose 6phosphate and 2-aminoethylphosphonic acid in successful trials (R-0-success), whereas Clostridium but not Roseburia was positively correlated with 2-aminoethylphosphonic acid in unsuccessful trials (R-0failure) (Fig. 5C). In recipients 1 day after FMT, Succinispira was positively correlated with taurine, which is linked to primary bile acid biosynthesis and $A B C$ transporters, in successful recipients (R-1-success) (Fig. 5C). Clostridium and Butyricicoccus were positively correlated with ribose 5-phosphate regardless of the success (R-1-success) and failure (R-1-failure) of FMT. In recipients, 7 days after FMT, Ruminococcus was negatively correlated with adenine, thymine, and 1,3-diaminoporopane in successful trials (R-7success) but positively correlated with adenine in unsuccessful trials (R-7-failure) (Fig. 5C). Although metabolites were correlated with changes in bacterial taxa, these results suggest that interactions may differ between successful and unsuccessful trials.

Weighted gene coexpression network analysis (WGCNA) of FMT outcome. If FMT is to be applied as a potential therapeutic for $\mathrm{CD}$ prevention, it is important to understand how microbiota and microbial products affect the incidence of $C D$ as well as the success or failure rate of FMT. Therefore, WGCNA [26] was performed to detect the possible inherent association among microbial taxa, clinical traits, and metabolites in both donors and recipients (Additional file 2: Fig. S13A). Major microbiota found in successful trials, e.g., Collinsella, Prevotella, Gemmiger, Acidaminococcus, and Selenomonas (Fig. 3), were mostly found in the gray module, which implies that this module is associated with FMT success. The microbial taxa responsible for each module are shown in Additional file 2: Fig. S13B and Additional file 3: Dataset S6. Concentrations of amino acids and organic acids were affected by both donor and 
recipient groups before and after FMT in both successful and unsuccessful trials (Fig. 4C, D); thus, WGCNA was individually extended to donors and recipients (day-0, day-1, and day-7) based on selected amino acids, major metabolites in TCA cycle, bile acids, and SCFAs. In donors, from the co-expression modules significantly associated with traits, modules 4 of 6 comprised taxa that were associated with several traits, e.g., an amino acid-, lactic acid-, and succinic acid-related module (MEred), a taurocholic acid-related module (MEblue and MEgreen), and a propionic acid-related module (MEturquoise). Major microbial taxa linked to the D-success group, especially Prevotella, Selenomonas, Succinispira, and Odoribacter, were positively correlated with the MEturquoise module (Fig. 6A; also shown in Additional file 2: Fig. S14 and Additional file 3: Dataset S7). For recipients, at day 0, four modules were formed based on the microbial taxa and traits of interest. MEblue was positively correlated with alanine, glycine, and cholic acid $(|r| \geq 0.5, P<0.05)$. The trait taurocholic acid was linked to MEblue and MEturquoise $(|r| \geq 0.5, P<$ 0.05) (Additional file 2: Fig. S15 and Additional file 3: Dataset S8). At day 1, microbial taxa were categorized into six modules (Additional file 2: Fig. S16A; Additional file 3: Dataset S9). Specifically, the genera Selenomonas and Acidaminococcus were positively correlated with MEbrown; for MEturquoise, the genus Sporobacter was positively correlated with succinic acid $(|r| \geq 0.5, \mathrm{P}<0.05)$ (Additional file 2: Fig. S16B). At day 7, microbial taxa were categorized into five modules (Fig. 6B; also shown in Additional file 2: Fig. S17 and Additional file 3: Dataset S10). In MEyellow, microbes such as Selenomonas, Lactobacillus, and Acidaminococcus, which were found in the R-7-success group (Fig. 3C), showed positive correlations with lactic acid and succinic acid (Fig. 6B; Additional file 2: Fig. S17). In contrast, Methanobrevibacter, Eggerthella, and Clostridium, all of which were linked to R-7-failure (Fig. 4; Additional file 2: Fig. S17), were significantly correlated with MEturquoise, which included the amino acids arginine, histidine, leucine, and phenylalanine (Fig. 6B). Thus, these microbial taxa could be possible predictive biomarkers for FMT failure in CD prevention.

Sporobacter is a potential biomarker of appropriate donors for FMT trials. To select optimal donors and diarrheal recipients for FMT, 158 diarrhea and non-diarrhea fecal samples were collected (Additional file 1: Table S4), and metagenomics analysis was performed to compare calves defined as donors and recipients in either successful or unsuccessful FMT trials. Diarrheal scores and microbial composition at the phylum level of calves are illustrated in Additional file 2: Fig. S18A, B. To select the potential predicators, e.g., specific microorganisms, for successful FMT trials, a principal coordinate analysis (PCOA) of the unweighted uniface distance matrix was performed on healthy and diarrheal calves (Fig. 7A). A significant difference was observed between healthy calves and unsuccessful, but not successful, donors in uniface distance analysis (Additional file 1: Table S5), suggesting that that the donors selected in FMT trials might be inappropriate in unsuccessful trials. Finally, an RF model was constructed to identify potential biomarkers in overall healthy and diarrheal calves along with calves recruited for FMT trials [29]. The Campylobacter, Actinobacillus, and Sporobacter were identified based on the mean decrease in accuracy; Campylobactor, Sporobacter, and Streptococcus were identified as the most discriminating predictors based on the mean decrease in gini criteria (Fig. 7B, C). Considering microbial abundance, Sporobacter was abundant in overall healthy and donor groups from successful FMT trials (Additional file 2: Fig. S18C). In contrast, Camphylobacter was found abundantly in the recipient diarrheal 
group following unsuccessful FMT trials (Additional file 2: Fig. S18D). Along with RF, LEfSe analysis was subsequently performed among these groups, in which Sporobacter was found to be differentially abundant in the healthy group (Fig. 7D). Taken together, these results suggest that Sporobacter may be a potential biomarker for the donors associated with FMT success.

\section{Discussion}

The success rate of FMT as a treatment for recurrent $C$. difficile infection in humans is extremely high at $>80 \%$, i.e., patients do not develop diarrhea and/or $C$. difficile is not detected in their feces at any time for 8 weeks posttreatment [37]. Recently, oral FMT was shown to be effective at treating multifactorial CD in multiple doses when compared with the effects of classical therapeutics such as antibiotics [6]. As CD has a substantial negative impact on the livestock industry, determining the efficacy of FMT in CD prevention and establishing the procedure as a practical therapeutic option is a priority. The aforementioned study [6] followed up the efficacy of FMT for 48 days; however, farmers have a notable tendency to overcome $\mathrm{CD}$ rapidly from a commercial farm management perspective. Therefore, we focused on changes in the intestinal environment of diarrheic calves during the first 7 days following a single rectal FMT. Consistent with the previous study, $70 \%$ of our trials were successful, as shown by dramatically decreasing diarrheal scores. In addition, we considered the $30 \%$ of unsuccessful trials with no changes in diarrheal score in an effort to identify the essential factors of FMT efficacy.

An important advance in the present study was the discovery of beneficial and non-beneficial bacteria identified from successful and unsuccessful trials. The genus Selenomonas, which utilizes lactic acid [38], was a bacterium that could be transferred from donors to recipients in successful (but not unsuccessful) trials within 7 days after FMT. The presence of Selenomonas in donors and recipients that have recovered from diarrhea is thought to be important for enhancing energy metabolism; several species of Selenomonas are known to be involved in ATP production through their effects on the succinate- propionate pathway [39]. WGCNA also confirmed the importance of Selenomonas, which was positively correlated with the amounts of lactic acid and succinic acid in donors and recipients 1 and 7 days after FMT in successful (but not unsuccessful) trials. Another important discovery was that of the genus Lactobacillus, which was abundant in recipients prior to FMT in successful (but not unsuccessful) trials. Lactobacillus promotes the growth of commensal microbiota and may develop a mutualistic relationship between the host and microbes in the gastrointestinal tract of successful recipients after FMT [40]. In contrast, the phylum Spirochaetes, which is responsible for mucohaemorrhagic colitis in swine [41], was abundant in unsuccessful donors and in unsuccessful recipients throughout the experiment even after FMT. Further studies are required to explore the role of Spirochaetes in the occurrence or progression of $C D$; nevertheless, these results suggest that this phylum may negatively affect the efficacy of FMT. Overall, Selenomonas, Lactobacillus, and Spirochaetes seem to be microorganisms associated with donors and/or recipients that lead to the success or failure of FMT; thus, a convenient strategy for detecting the presence of such bacteria in the field should be established to increase the rate of FMT success. 
Gene expression associated with ribosomal translation, amino acid metabolism, and carbohydrate metabolism is reduced in calves suffering from hemorrhagic diarrhea $[3,6,13]$; therefore, it is important that the key microbial clusters for regulating intestinal metabolites are investigated in FMT studies. Altered amino acid metabolism has been also observed in cats and dogs suffering from chronic diarrhea $[42,43]$; consequently, amino acid changes may be a factor in the remission of CD. Consistent with a previous study [6], we observed decreased levels of most fecal amino acids in the successful recipients 7 days after FMT (compared with the original levels prior to therapy). In contrast, the fecal amino acid levels mostly increased after FMT in unsuccessful recipients. Furthermore, the significant microbial taxa of the R-7-failure group (e.g., the family Bacillaceae and genera Methanobrevibacter, Eggerthella, and Clostridium) were positively correlated with the levels of branched-chain amino acids (i.e., leucine and valine) as well as with arginine, histidine, phenylalanine, and tryptophan. Given that the amino acids in feces are positively correlated with the severity of Crohn's disease [44], high amounts of free amino acids suggest that suboptimal nutrient utilization occurred due to dysbiosis in unsuccessful FMT trials. The metabolic milieu generated in unsuccessful trials may help to regulate the colonization and pathogenicity of harmful microbiota. For example, biogenic amino acids such as isoamylamine were identified as potential markers in unsuccessful trials. Previously, isoamylamine was shown to play a specific role in the encystation and differentiation processes of pathogenic protozoa, e.g., Entamoeba, Giardia, Acanthamoeba, and Balamuthia, and to exhibit a distinct kinetics feature of their survival [45]. Dihydroxyacetone phosphate and glycerol-3-phosphate, which had the highest VIP scores in unsuccessful donors, are dominantly produced by the flagellated protozoan Giardia duodenalisis, a worldwide parasite that causes giardiasis (an acute and chronic diarrheal disease) [46]. To ensure the success of FMT, it may be necessary, therefore, to also ensure the absence of metabolites associated with unsuccessful FMT in both donors and recipients prior to treatment.

Notably, the establishment of FMT as a veterinary practice for effective treatment of CD remains difficult due to safety concerns regarding the transmission of virulence factors [47]. It is also difficult to determine the potential of successful donors prior to FMT; however, a machine-learning approach has provided some predictions of the microbial composition required in potential donors [48]. We identified Sporobacter as a potential biomarker for donor selection using RF classification and LEfSe analysis; Sporobacter was also positively correlated with the production of succinic acid in R-1-success, suggesting that it may play an important role in the interactions of microbiota and metabolites in potential donors. Establishing the discriminative characteristics using novel prediction model in this study for identifying calf with diarrheal incidences who are at high risk of failure even after FMT therapy may assist the veterinary physician in subsequent management plans. Thus, our study emphasizes the use of a machine-learning approach to help protect livestock. Further studies are needed to determine whether the donor FMT itself induces the complete resolution of CD or whether it helps promote a spontaneous recovery. Indeed, it is important to develop a robust understanding of the gut microbiome and metabolome across individuals in general. This study was the first to examine multidonor FMT as a strategy for $\mathrm{CD}$ treatment through the remediation of gut microbiota with an emphasis on the personalization of interventions; additionally, the inherent individualized variability in microbiota, 
metabolites, and other physiological features was considered. Our findings provide new insights into developing successful FMT strategies with a multiomics approach and they may help to advance research into microbial therapeutics for $C D$ treatment.

\section{Conclusion}

In this study, we demonstrated that fecal microbiota transplantation (FMT) is an effective treatment option for the prevention of diarrhea in calves, specifically by identifying a beneficial microbial cluster as well as functional metabolites. We showed that FMT success or failure depends on the microbial composition of both donors and recipients. We also identified the microbial genera Sporobacter and Selenomonas in donor calves and Lactobacillus in recipient diarrheal calves in successful FMT cases; thus, the presence of these genera may predict FMT success. These findings have enormous significance for the livestock industry because FMT could eventually address the challenge of calf diarrhea prevention as well as the use of excessive antibiotics.

\section{Declarations}

\section{Ethics approval and consent to participate}

The study was conducted in accordance with the Guidelines for Laboratory Animal Welfare and Animal Experiment Control promulgated by the ethics committee of Chiba NOSAI Kanji-kai (Approval No. CNS190901 and CNS190902).

\section{Consent for publication}

Not applicable

\section{Availability of data and material:}

All data generated or analyzed during this study are included in this published article [and its supplementary information files]. However, further data can be available on request to corresponding author.

\section{Competing Interest Statement:}

The authors declare no competing interest.

\section{Funding}

This study was mostly supported by Livestock Promotional Subsidy from the Japan Racing Association (to TN), and also supported by Grants-in-Aid for Scientific Research (A) 18H03969 (to TN), Grants-in-Aid for Early-Career Scientists 20K15478 (to JI), and Core-to-Core Program (Advanced Research Networks) (to 
TN), the Grant for Joint Research Project of the Institute of Medical Science, The University of Tokyo (to $\mathrm{TN})$.

\section{Authors' contributions}

J.I. H.T. and T.N. designed research, M.T., Y.S., Y.G., N.O., K.S., E.T., E.M., T.S., and C.U. performed FMT, J.I., A.M., Y.Su, Y.Sa., M.F., and K.U. analyzed samples, J.I. analyzed data, H.Y. and H.A. discussed the data, J.I., H.T., and T.N. wrote the paper.

\section{Acknowledgements}

We thank Drs. Hidetoshi Kato, Takafumi Goto and Jun Kunisawa for practical advice for this study.

\section{References}

1. Khoruts A, Sadowsky MJ. Understanding the mechanisms of faecal microbiota transplantation. Nat Rev Gastroenterol Hepatol. 2016;13:508-16.

2. Zhang F, Cui B, He X, Nie Y, Wu K, Fan D. Microbiota transplantation: concept, methodology and strategy for its modernization. Protein Cell. 2018;9:462-73.

3. Gomez DE, Arroyo LG, Costa MC, Viel L, Weese JS. Characterization of the Fecal Bacterial Microbiota of Healthy and Diarrheic Dairy Calves. J Vet Intern Med. 2017;31:928-39.

4. Li SS, Zhu A, Benes V, Costea PI, Hercog R, Hildebrand F, et al. Durable coexistence of donor and recipient strains after fecal microbiota transplantation. Science. 2016;352:586-9.

5. Xiao Y, Angulo MT, Lao S, Weiss ST, Liu Y-Y. An ecological framework to understand the efficacy of fecal microbiota transplantation. Nat Commun. 2020;11:1-17.

6. Kim HS, Whon TW, Sung H, Jeong Y-S, Jung ES, Shin N-R, et al. Longitudinal evaluation of fecal microbiota transplantation for ameliorating calf diarrhea and improving growth performance. Nat Commun. 2021;12:1-16.

7. Huang MZ, Cui DA, Wu XH, Hui W, Yan ZT, Ding XZ, et al. Serum Metabolomics Revealed the Differential Metabolic Pathway in Calves with Severe Clinical Diarrhea Symptoms. Animals (Basel) 2020;10.

8. Cho Y-i, Yoon K-J. An overview of calf diarrhea-infectious etiology, diagnosis, and intervention. J Vet Sci. 2014;15:1-17.

9. Foster D, Smith GW. Pathophysiology of diarrhea in calves. Vet Clin North Am Food Anim Pract. 2009;25:13-36.

10. Meganck V, Hoflack G, Opsomer G. Advances in prevention and therapy of neonatal dairy calf diarrhoea: a systematical review with emphasis on colostrum management and fluid therapy. Acta Vet Scand. 2014;56:1-8.

11. Constable PD. Treatment of calf diarrhea: antimicrobial and ancillary treatments. Vet Clin North Am Food Anim Pract. 2009;25:101-20. 
12. Hu J, Ma L, Nie Y, Chen J, Zheng W, Wang X, et al. A microbiota-derived bacteriocin targets the host to confer diarrhea resistance in early-weaned piglets. Cell Host Microbe. 2018;24:817-32.e8.

13. Zeineldin $M$, Aldridge B, Lowe J. Dysbiosis of the fecal microbiota in feedlot cattle with hemorrhagic diarrhea. Microb Pathog. 2018;115:123-30.

14. Smillie CS, Sauk J, Gevers D, Friedman J, Sung J, Youngster I, et al. Strain tracking reveals the determinants of bacterial engraftment in the human gut following fecal microbiota transplantation. Cell Host Microbe. 2018;23:229-40.e5.

15. Duvallet C, Zellmer C, Panchal P, Budree S, Osman M, Alm E. Framework for rational donor selection in fecal microbiota transplant clinical trials. PloS One. 2019;14:e0222881.

16. Roguet A, Eren AM, Newton RJ, McLellan SL. Fecal source identification using random forest. Microbiome. 2018;6:1-15.

17. Topçuoğlu BD, Lesniak NA, Ruffın IVMT, Wiens J, Schloss PD. A framework for effective application of machine learning to microbiome-based classification problems. mBio. 2020;11:e00434-00420.

18. Lin X, Liu X, Xu J, Cheng K-K, Cao J, Liu T, et al. Metabolomics analysis of herb-partitioned moxibustion treatment on rats with diarrhea-predominant irritable bowel syndrome. Chin Med. 2019;14:18.

19. Nochi T, Suzuki S, Ito S, Morita S, Furukawa M, Fuchimoto D, et al. Elucidation of the Effects of a Current X-SCID Therapy on Intestinal Lymphoid Organogenesis Using an In Vivo Animal Model. Cell Mol Gastroenterol Hepatol. 2020;10:83-100.

20. Bolyen E, Rideout JR, Dillon MR, Bokulich NA, Abnet CC, Al-Ghalith GA, et al. Reproducible, interactive, scalable and extensible microbiome data science using QIIME 2. Nat Biotechnol. 2019;37:852-7.

21. McDonald D, Price MN, Goodrich J, Nawrocki EP, DeSantis TZ, Probst A, et al. An improved Greengenes taxonomy with explicit ranks for ecological and evolutionary analyses of bacteria and archaea. ISME J. 2012;6:610-8.

22. Segata N, Izard J, Waldron L, Gevers D, Miropolsky L, Garrett WS, et al. Metagenomic biomarker discovery and explanation. Genome Biol. 2011;12:1-18.

23. Dhariwal A, Chong J, Habib S, King IL, Agellon LB, Xia J. MicrobiomeAnalyst: a web-based tool for comprehensive statistical, visual and meta-analysis of microbiome data. Nucleic Acids Res. 2017;45:W180-8.

24. Iwai S, Weinmaier T, Schmidt BL, Albertson DG, Poloso NJ, Dabbagh K, et al. Piphillin: improved prediction of metagenomic content by direct inference from human microbiomes. Plos One. 2016;11:e0166104.

25. Sugiura Y, Taguchi R, Setou M. Visualization of spatiotemporal energy dynamics of hippocampal neurons by mass spectrometry during a kainate-induced seizure. PLoS One. 2011;6:e17952.

26. Langfelder P, Horvath S. WGCNA: an R package for weighted correlation network analysis. BMC Bioinformatics. 2008;9:559. 
27. Chong J, Wishart DS, Xia J. Using MetaboAnalyst 4.0 for comprehensive and integrative metabolomics data analysis. Curr Protoc Bioinformatics. 2019;68:e86.

28. Benjamini Y, Hochberg Y. Controlling the false discovery rate: a practical and powerful approach to multiple testing. J R Stat Soc Series B Stat Methodol. 1995;57:289-300.

29. Liaw A, Wiener M. Classification and regression by randomForest. R News. 2002;2:18-22.

30. Bozukluhan K, Merhan O, Gokce H, Deveci H, Gokce G, Ogun M, et al. Alterations in lipid profile in neonatal calves affected by diarrhea. Vet World. 2017;10:786.

31. Negri Filho LC, Pereira CES, Chineze PHN, Bogado ALG, Bronkhorst DE, Lunardi M, et al. Use of the enzyme gamma-glutamyl transferase (GGT) as an indirect measure of passive transfer of immunity in holstein calves and association with the occurrence of diarrhea after birth. Biosci J 2016;32.

32. Wang Z, Wang Q, Zhao J, Gong L, Zhang Y, Wang X, et al. Altered diversity and composition of the gut microbiome in patients with cervical cancer. AMB Express. 2019;9:1-9.

33. Dulski T, Kujawa R, Godzieba M, Ciesielski S. Effect of Salinity on the Gut Microbiome of Pike Fry (Esox lucius). Appl Sci. 2020;10:2506.

34. Cho H-W, Kim SB, Jeong MK, Park Y, Miller N, Ziegler T, et al. Discovery of metabolite features for the modelling and analysis of high-resolution NMR spectra. Int J Data Min Bioinform. 2008;2:176-92.

35. McHardy IH, Goudarzi M, Tong M, Ruegger PM, Schwager E, Weger JR, et al. Integrative analysis of the microbiome and metabolome of the human intestinal mucosal surface reveals exquisite interrelationships. Microbiome. 2013;1:1-19.

36. Oksanen J, Kindt R, Legendre P, O'Hara B, Stevens MHH, Oksanen MJ, et al. The vegan package. Community ecology package. 2007;10:719.

37. Allegretti JR, Allegretti AS, Phelps E, Xu H, Fischer M, Kassam Z. Classifying Fecal Microbiota Transplantation Failure: An Observational Study Examining Timing and Characteristics of Fecal Microbiota Transplantation Failures. Clin Gastroenterol Hepatol. 2017;16:1832-3.

38. Evans JD, Martin SA. Factors affecting lactate and malate utilization by Selenomonas ruminantium. Appl Environ Microbiol. 1997;63:4853-8.

39. Hall MB, Weimer P. Divergent utilization patterns of grass fructan, inulin, and other nonfiber carbohydrates by ruminal microbes. J Dairy Sci. 2016;99:245-57.

40. Ekmekciu I, von Klitzing E, Neumann C, Bacher P, Scheffold A, Bereswill S, et al. Fecal microbiota transplantation, commensal Escherichia coli and Lactobacillus johnsonii strains differentially restore intestinal and systemic adaptive immune cell populations following broad-spectrum antibiotic treatment. Front Microbiol. 2017;8:2430.

41. Rubin JE, Costa MO, Hill JE, Kittrell HE, Fernando C, Huang Y, et al. Reproduction of mucohaemorrhagic diarrhea and colitis indistinguishable from swine dysentery following experimental inoculation with "Brachyspira hampsonii" strain 30446. PloS One. 2013;8:e57146.

42. Minamoto Y, Otoni CC, Steelman SM, Büyükleblebici O, Steiner JM, Jergens AE, et al. Alteration of the fecal microbiota and serum metabolite profiles in dogs with idiopathic inflammatory bowel disease. 
Gut Microbes. 2015;6:33-47.

43. Suchodolski JS, Foster ML, Sohail MU, Leutenegger C, Queen EV, Steiner JM, et al. The fecal microbiome in cats with diarrhea. PloS One. 2015;10:e0127378.

44. Battaglioli EJ, Hale VL, Chen J, Jeraldo P, Ruiz-Mojica C, Schmidt BA, et al. Clostridioides difficile uses amino acids associated with gut microbial dysbiosis in a subset of patients with diarrhea. Sci Transl Med. 2018;10.

45. Jeelani G, Sato D, Husain A, Escueta-de Cadiz A, Sugimoto M, Soga T, et al. Metabolic profiling of the protozoan parasite Entamoeba invadens revealed activation of unpredicted pathway during encystation. PLoS One. 2012;7:e37740.

46. Lalle M, Camerini S, Cecchetti S, Finelli R, Sferra G, Müller J, et al. The FAD-dependent glycerol-3phosphate dehydrogenase of Giardia duodenalis: an unconventional enzyme that interacts with the g14-3-3 and it is a target of the antitumoral compound NDBHEX. Front Microbiol. 2015;6:544.

47. Chu ND, Crothers JW, Nguyen LT, Kearney SM, Smith MB, Kassam Z, et al. Dynamic colonization of microbes and their functions after fecal microbiota transplantation for inflammatory bowel disease. mBio. 2019:649384.

48. de Leeuw MA, Duval MX. Selecting donors for faecal microbiota transplantation in ulcerative colitis. medRxiv. 2020.

\section{Figures}


A
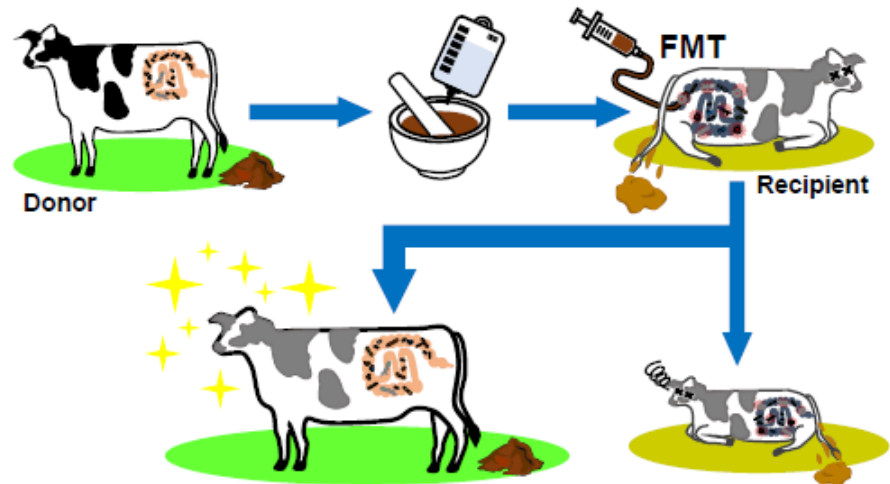

Successful

B
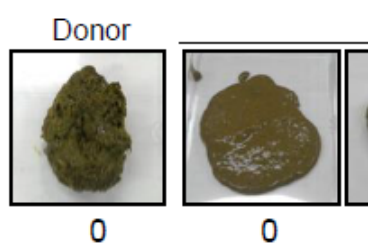

Recipient

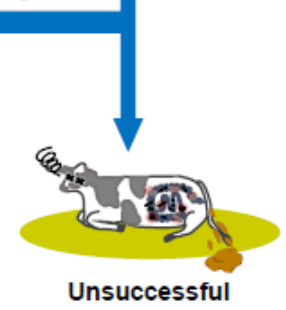

Unsuccessful
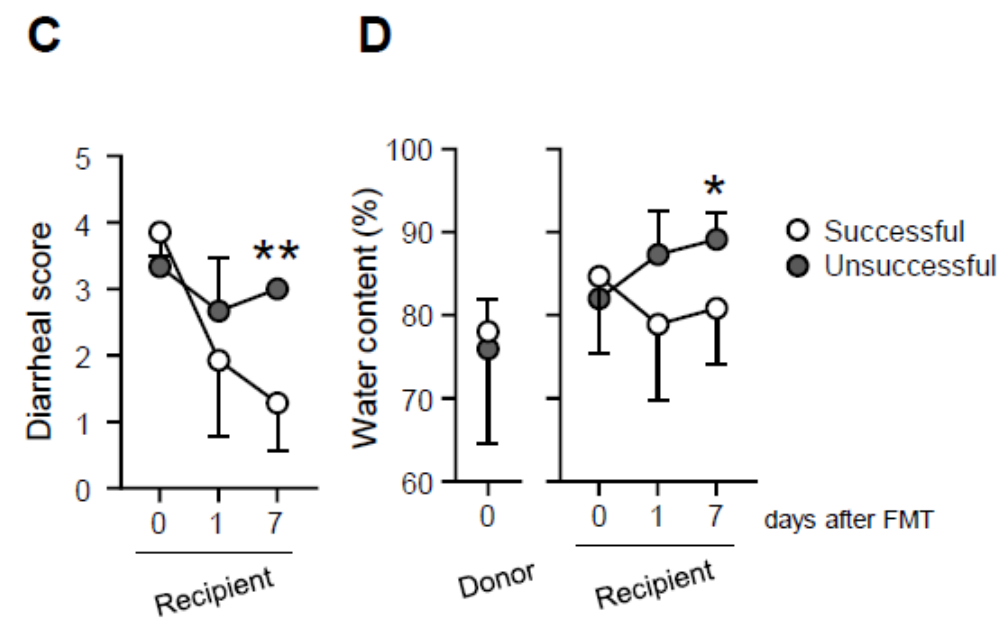

$\mathbf{F}$

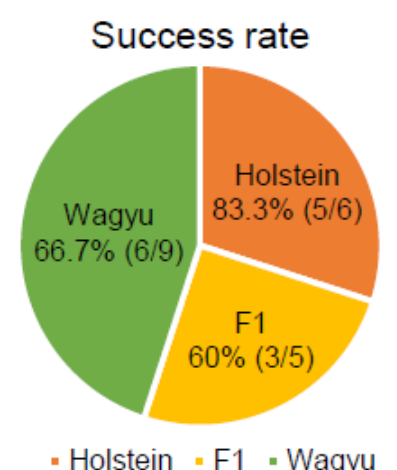

- Holstein " F1 - Wagyu
$\mathbf{E}$

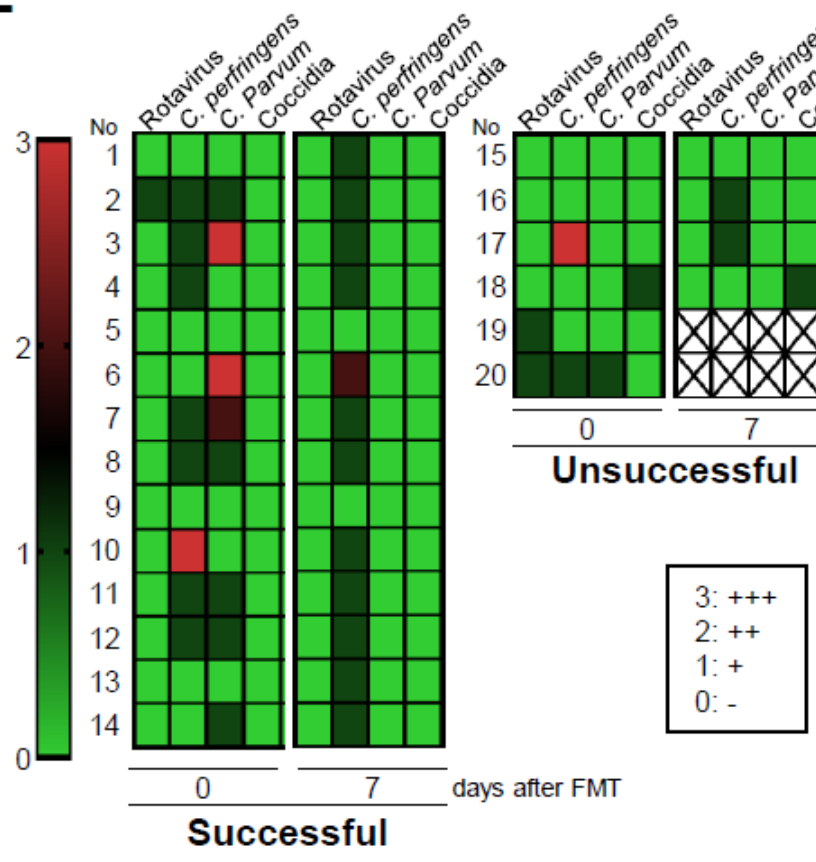

\section{G}

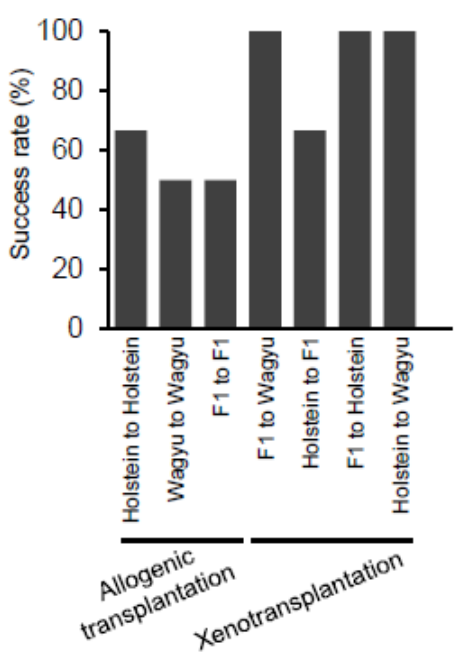

Figure 1

Efficacy of FMT in CD prevention. (A) Graphical design of the study, (B) images of feces of healthy donor and recipient diarrheal calves in successful trials, (C) diarrheal score, (D) fecal water content, (E) FMTspecific pathogen detection, and success rate based on $(F)$ cow strain and $(G)$ allogenic and xenotransplantation. Donor $(n=14)$, recipient $0(n=14)$, recipient $1(n=14)$, and recipient $7(n=14)$ in successful trials; donor $(n=6)$, recipient $0(n=6)$, recipient $1(n=6)$, and for recipient $7(n=6)$ in unsuccessful trials. "+++" 109 Cfu f/feces; "++" 108 Cfu g/feces; and "+" 107 Cfu g/feces. *P< 0.05 and $\star * P<0.01$ (Student's t-test). 
A

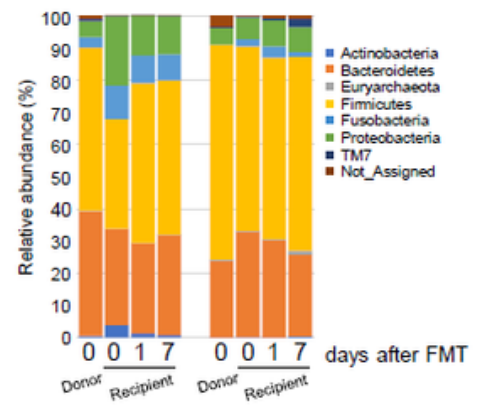

Successful Unsuccessful
B

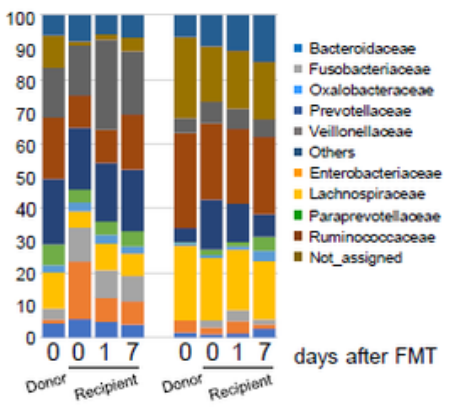

Successful Unsuccessful
C

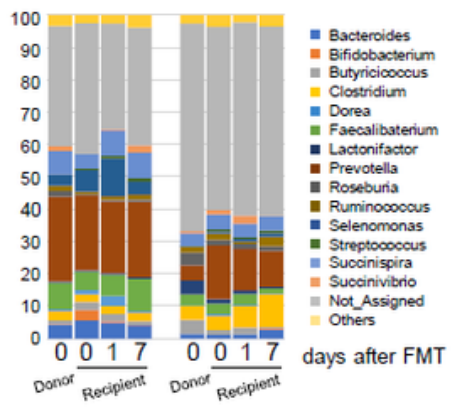

Successful Unsuccessful

D
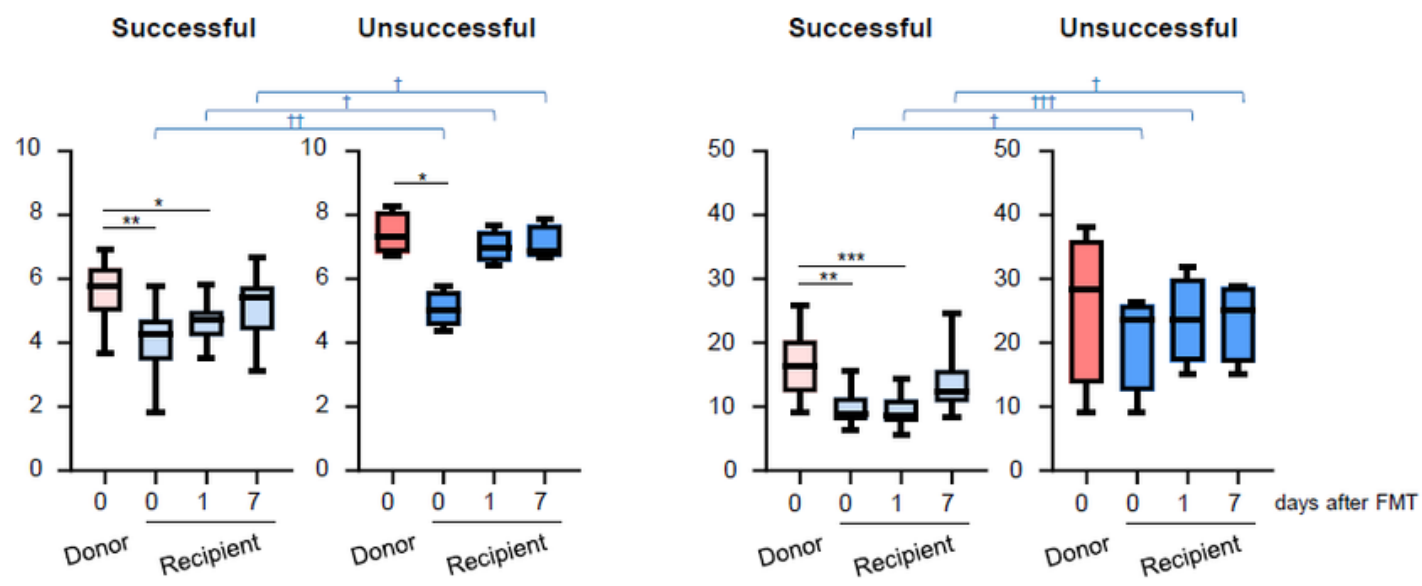

E

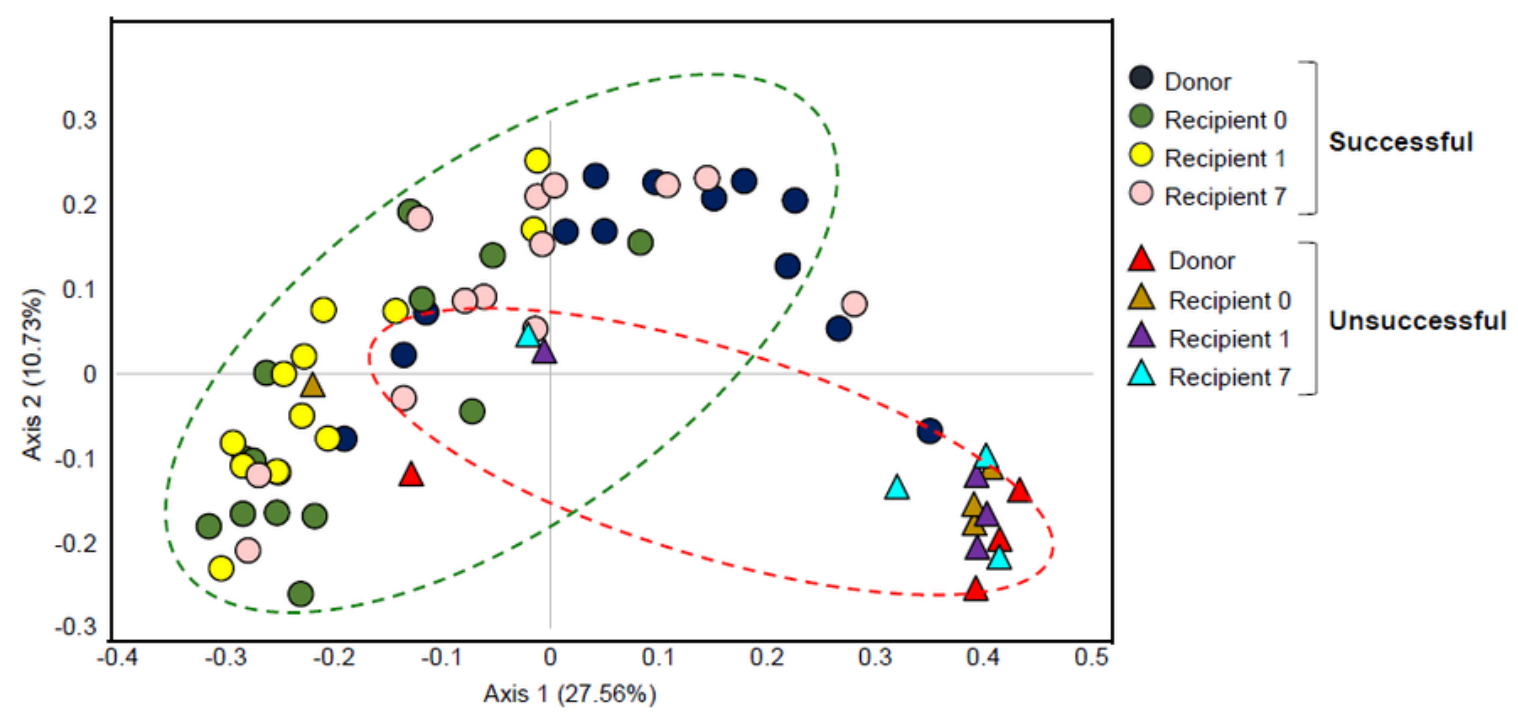

\section{Figure 2}

Microbial composition in the FMT study. Microbial composition at the (A) phylum, (B) family, and (C) genus levels. Donor $(n=13)$, recipient $0(n=13)$, recipient $1(n=13)$, and recipient $7(n=13)$ in successful trials; donor $(n=4)$, recipient $0(n=4)$, recipient $1(n=4)$, and recipient $7(n=4)$ in unsuccessful trials. (D) Alpha diversity index based on Shannon's index and faith phylogenetic diversity; (E) PCoA based on the unweighted distance matrix of the bacterial 16S rRNA gene sequence data for fecal samples (donor and 
recipient $n$ numbers in successful and unsuccessful trials as shown above). $P$ values ( ${ }^{* \star *} \mathrm{P}<0.001,{ }^{\star} \mathrm{P}<$ 0.01 , and $* P<0.05$ ) indicate statistical significance either successful or unsuccessful trial by one-way ANOVA followed by tukey multiple comparison test. $P$ values $(+\uparrow P<0.01,+P<0.05)$ indicate statistical significance by Student's t-test.
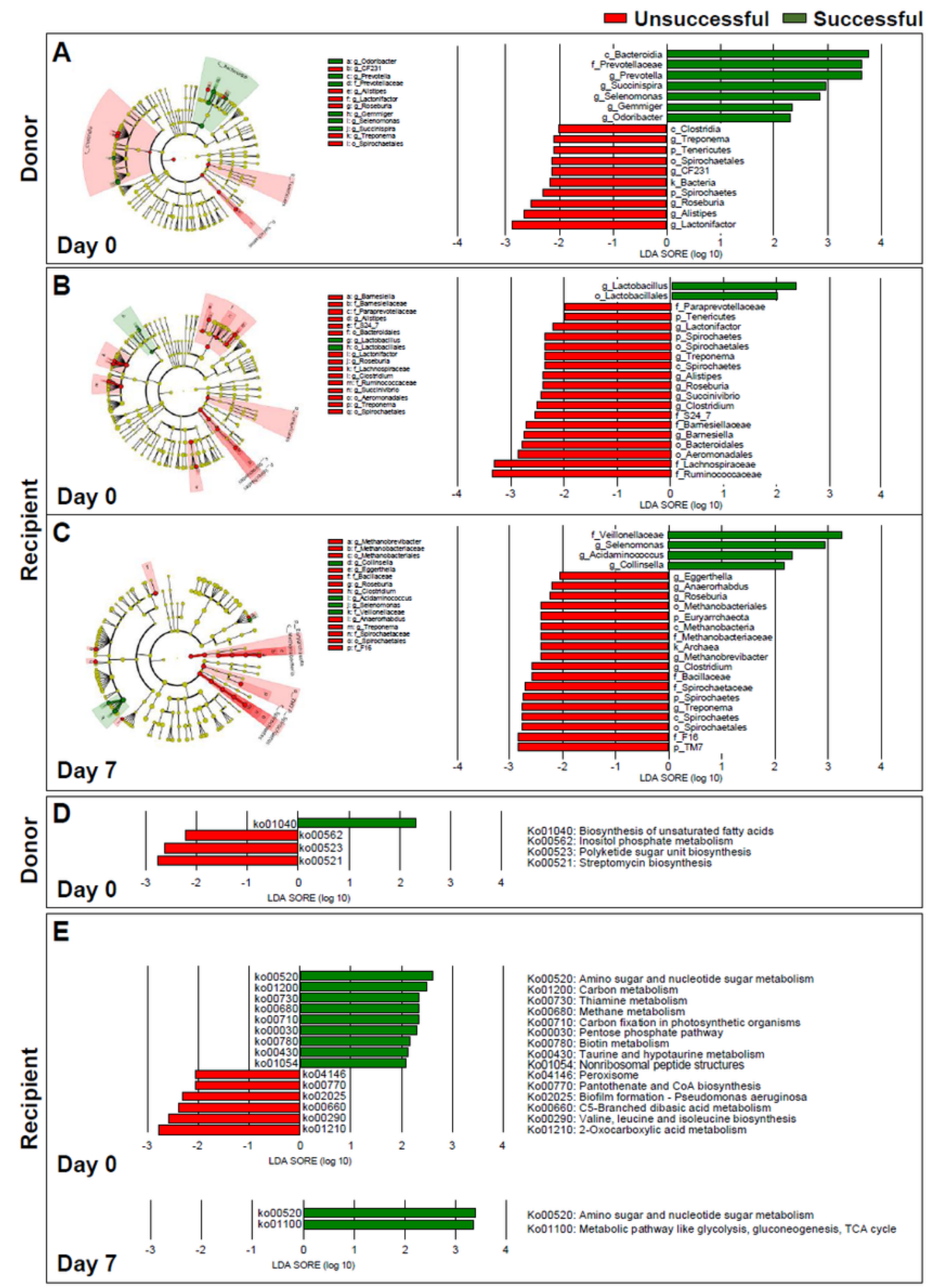

Figure 3 
Identification of differentially abundant microbiota. (A-C) Cladogram [phylum (p), class (c), order (o), family (f), genera (g)] and linear discriminant analysis (LDA) scores of differentially abundant taxa in the donor (day 0$)(A)$, recipient day $0(B)$, and recipient day $7(C)$ [donor $(n=13)$, recipient $0(n=13)$, recipient $1(n=13)$, and recipient $7(n=13)$ in successful trials; donor $(n=4)$, recipient $0(n=4)$, recipient $1(n=4)$, and for recipient $7(n=4)$ in unsuccessful trials]. Predicted differentially expressed KEGG metabolic pathways in donors (D) and recipients (E) in successful and unsuccessful trials identified by Piphillin and linear discriminant analysis (LDA $>2.0, \mathrm{P}<0.05)$. Data shown for successful and unsuccessful trials for donors and recipients ( $\mathrm{n}$ numbers as shown above). 
A

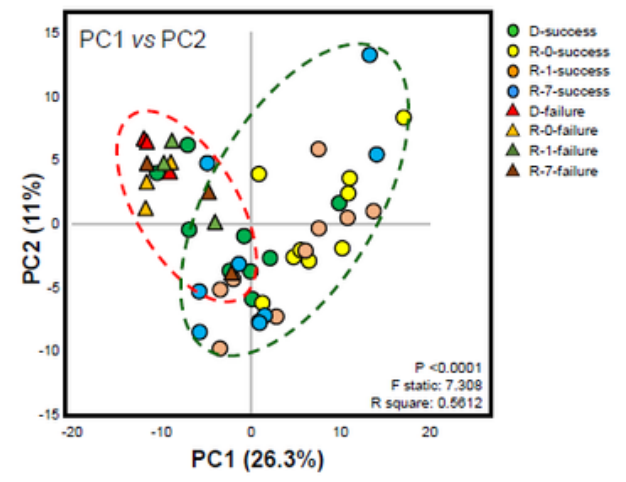

B

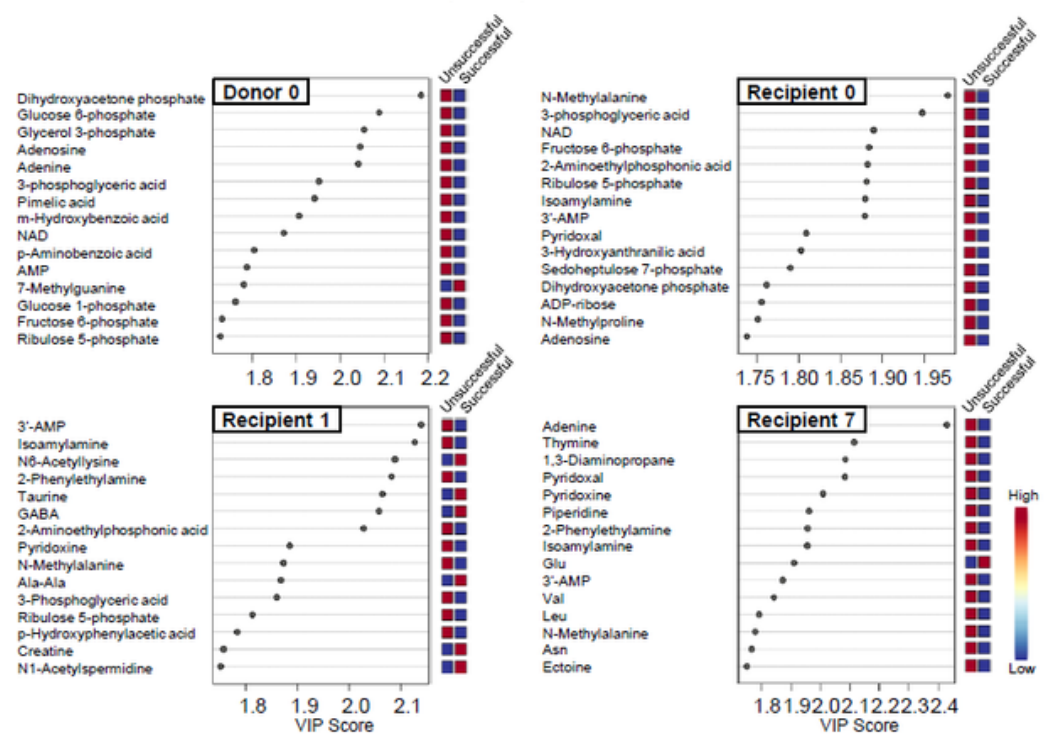

C

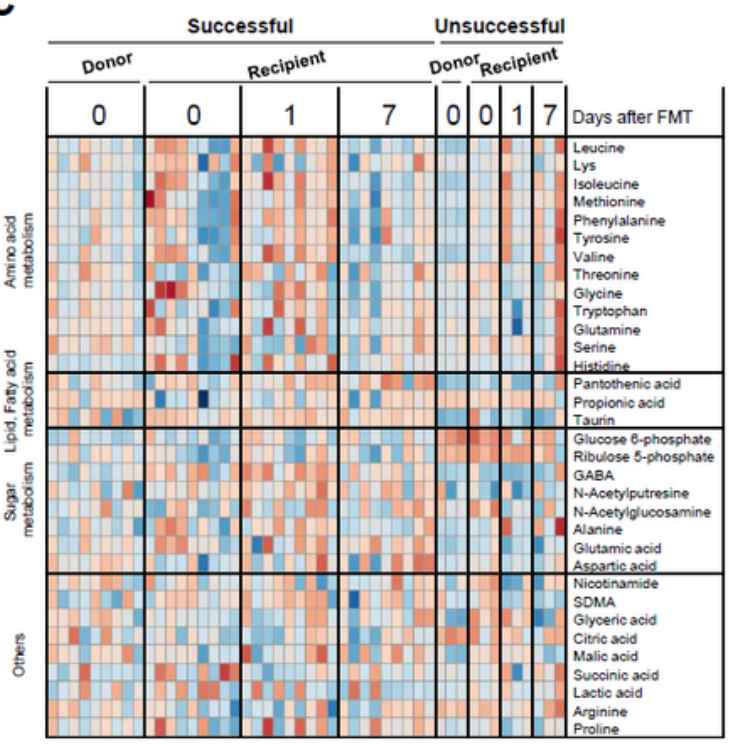

D

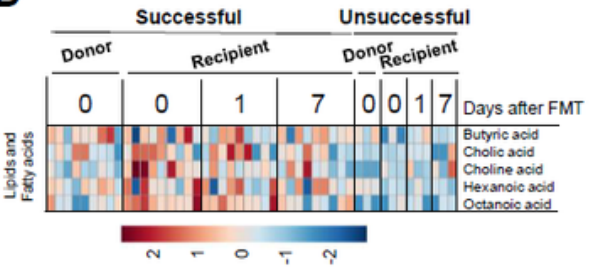

\section{Figure 4}

Multivariate unsupervised principal component analysis for the metabolomes of the calves' fecal samples. Data were obtained using CE-TOFMS analysis for donor $(n=9)$, recipient $0(n=9)$, recipient $1(n$ $=9)$, and recipient $7(n=9)$ in successful trials, and for donor $(n=3)$, recipient $0(n=3)$, recipient $1(n=3)$, and recipient $7(n=3)$ in unsuccessful trials. (A) Fecal metabolomics analysis to identify potential biomarkers. (B) Variables importance in projection (VIP) scores obtained from partial least squares 
discriminant analysis for donor (at day 0 ) and recipients (on days 0,1 , and 7). (C-D) Heatmaps based on the euclidean distance of the major metabolites responsible for amino acid metabolism, lipid and fatty acid metabolism, sugar metabolism, and others in both successful and unsuccessful trials. Major metabolites for lipid and fatty acid metabolism are based on relative area. Each colored cell on the map corresponds to a concentration value, with samples shown in rows and metabolites in columns (donor and recipient $n$ numbers in successful and unsuccessful trials as shown above).

A

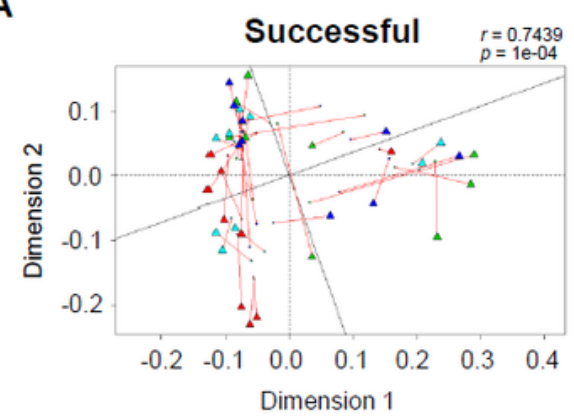

C

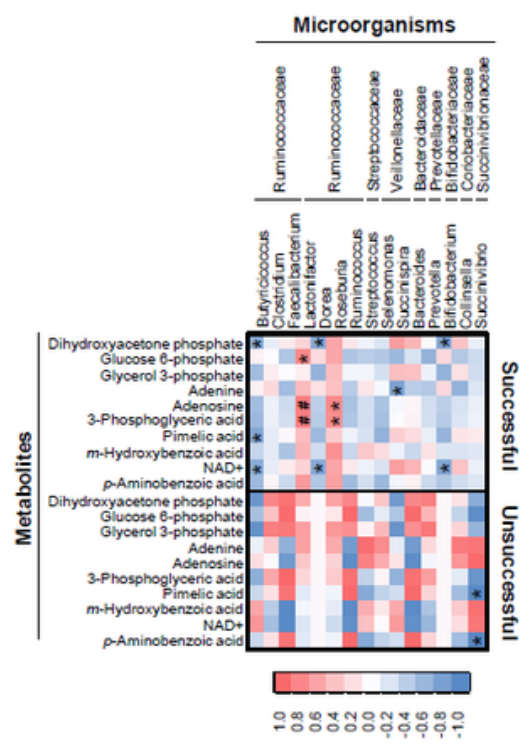

Donor
B
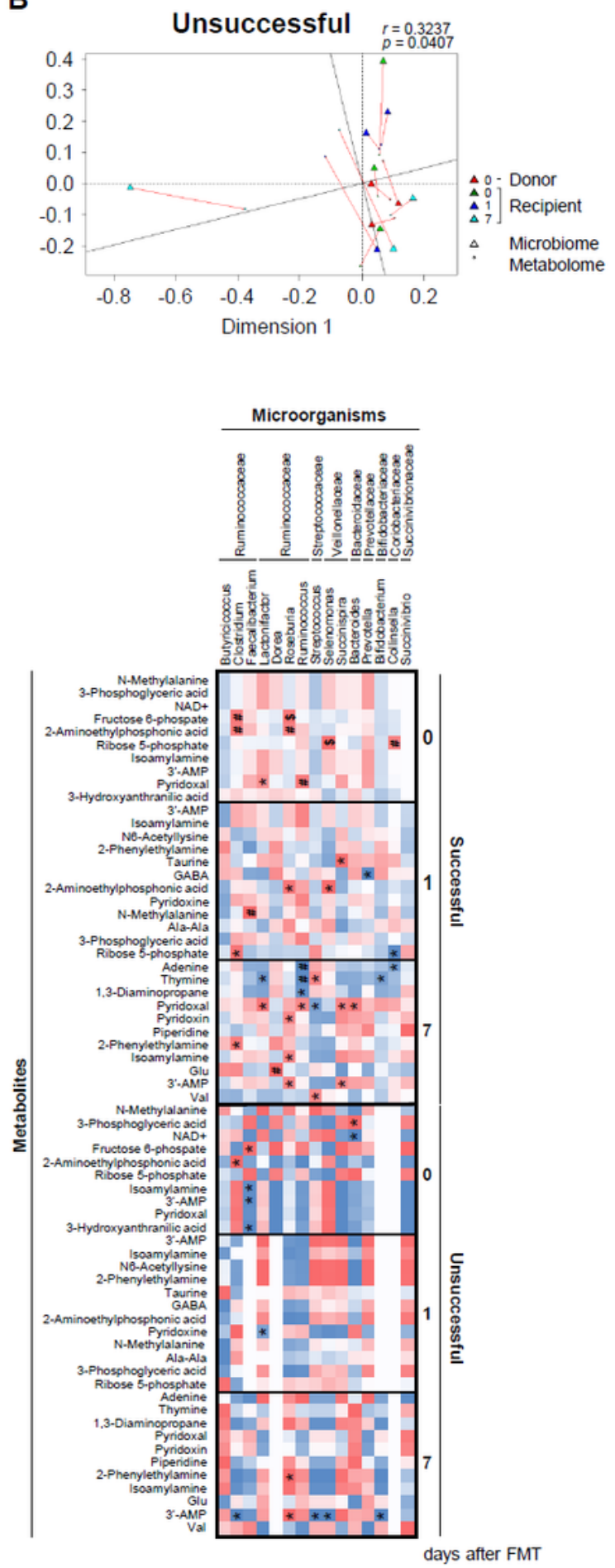

Recipient 
Procrustes analysis for correlations between microbiota and metabolites. Longer lines on the Procrustes plot specify more within-subject dissimilarity. (A) Overall successful trial (Procrustes sum of squares: 0.4467; correlation: 0.7439; $P=0.0001)$. (B) Overall unsuccessful trials (Procrustes sum of squares: 0.611; correlation: 0.6237; $P=0.0407)$. Data are shown for donor $(n=9)$, recipient $0(n=9)$, recipient $1(n$ $=9)$, and recipient $7(n=9)$ in successful trials, and for donor $(n=3)$, recipient $0(n=3)$, recipient $1(n=3)$, and recipient $7(n=3)$ in unsuccessful trials. (C) Pearson correlations in donor (D-success and D-failure), and (B) for the recipient group at days 0,1 , and 7 (donor and recipient $n$ numbers, as shown above for successful and unsuccessful trials). Color shows Pearson correlation coefficient distribution: blue represents a significant negative correlation $(P<0.05)$, red represents a significant positive correlation $(P$ $<0.05)$, and white represents a non-significant correlation $(P \geq 0.05)$. $\$ P<0.001, \# P<0.01$, and $* P<0.05$ indicate statistical significance. 
A

Module-trait relationship

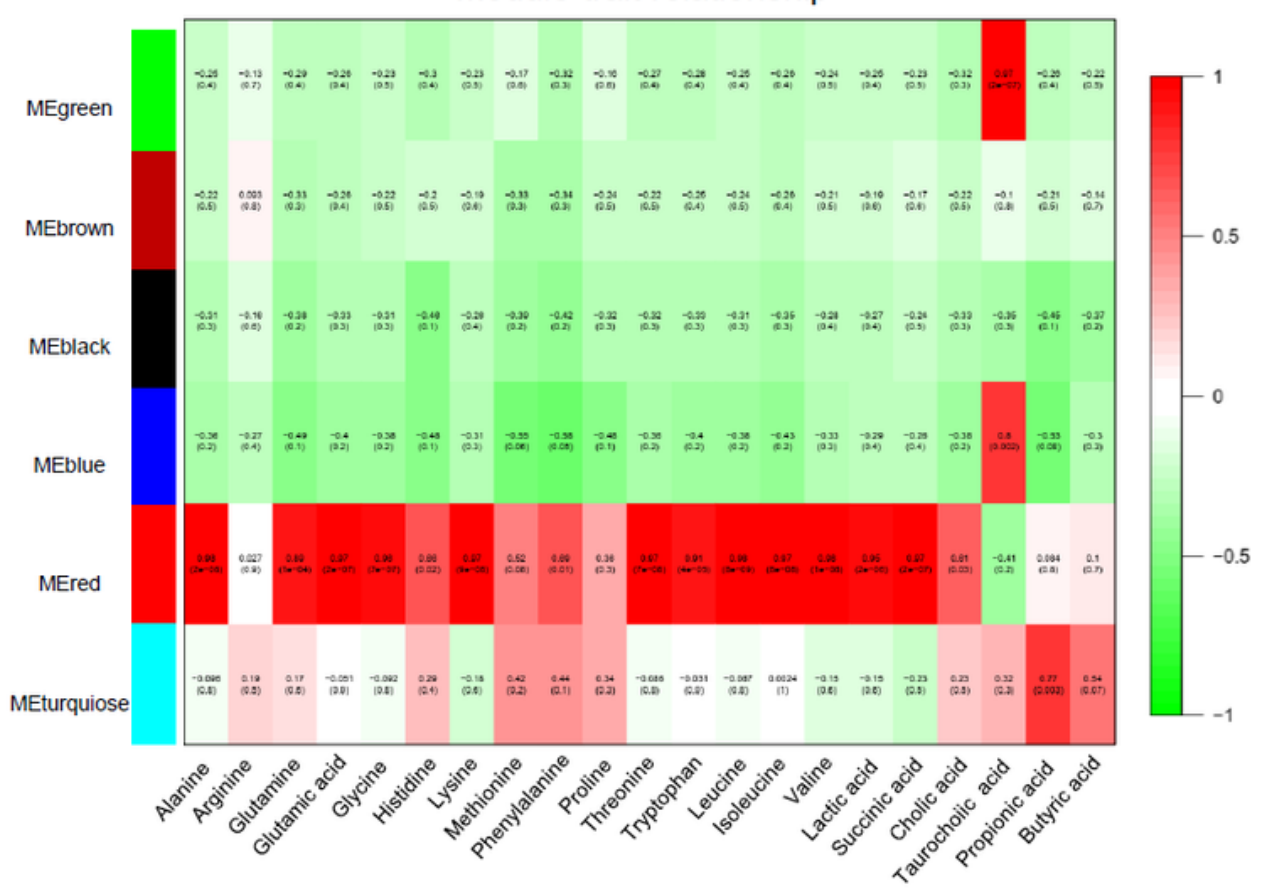

B

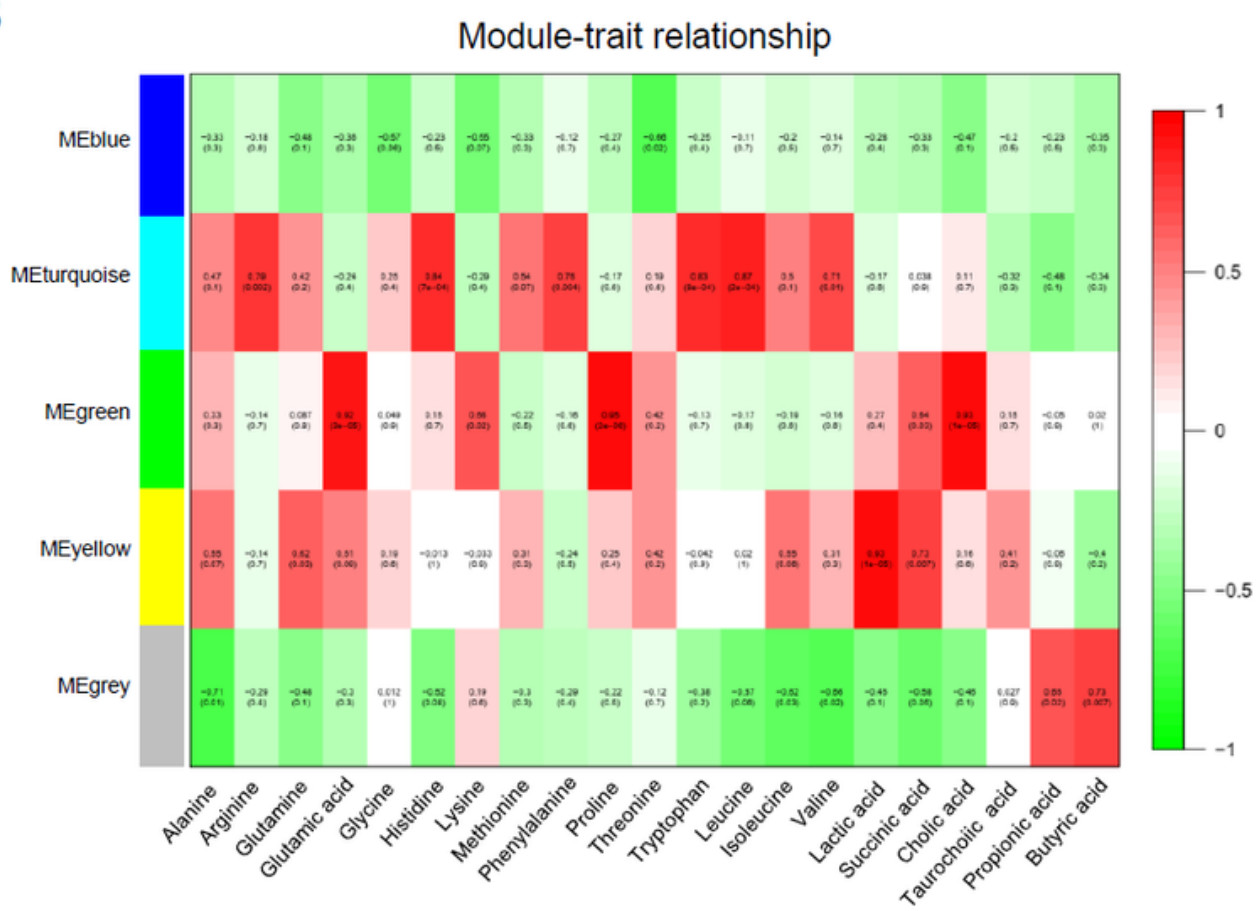

\section{Figure 6}

Weighted gene coexpression network analysis for microbiota and metabolites. Module-trait associations based on (A) donor groups for donors $(n=9)$ in successful trials and donors $(n=3)$ in unsuccessful trials. (B) Recipients at day 7 for recipients $(n=9)$ in successful trial and recipients $(n=3)$ in unsuccessful trials. Each row corresponds to a module. Each column corresponds to a phenotypic trait (labeled below column). Each cell at the row-column intersection contains the correlation coefficient and 
p-value (in brackets) for the module and the corresponding trait. A highly positive correlation between a specific module and a trait is indicated by dark red, whereas a negative correlation is indicated in green.

A

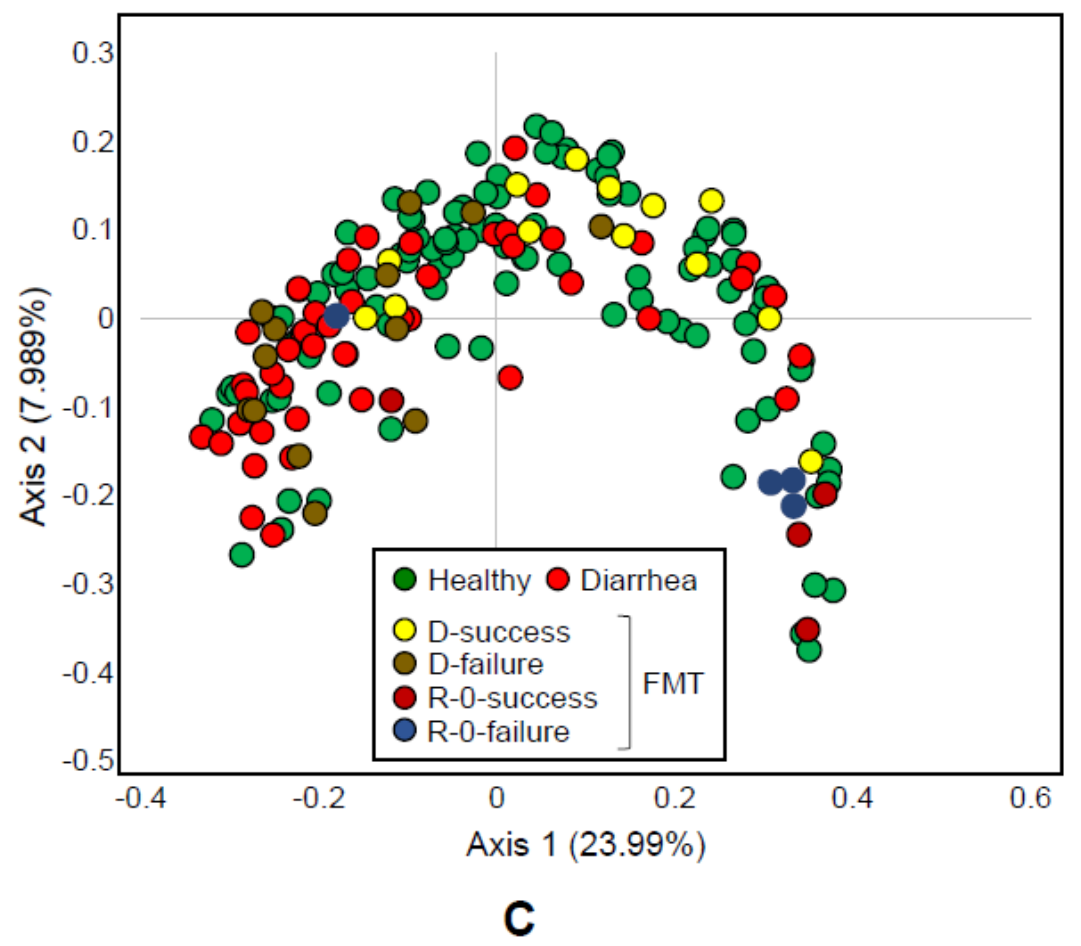

B

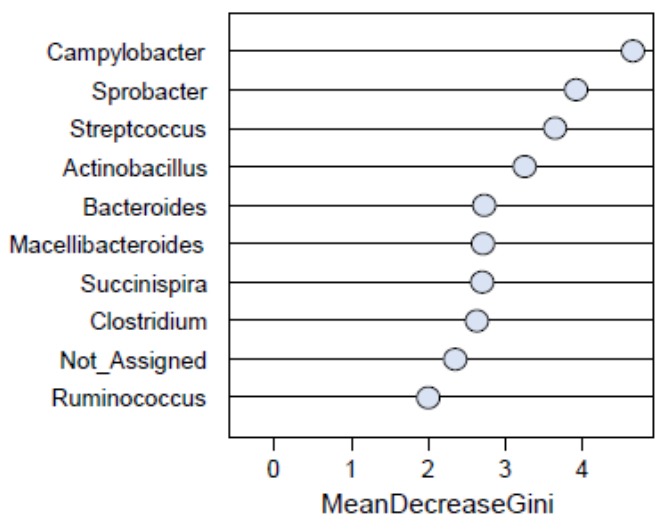

D
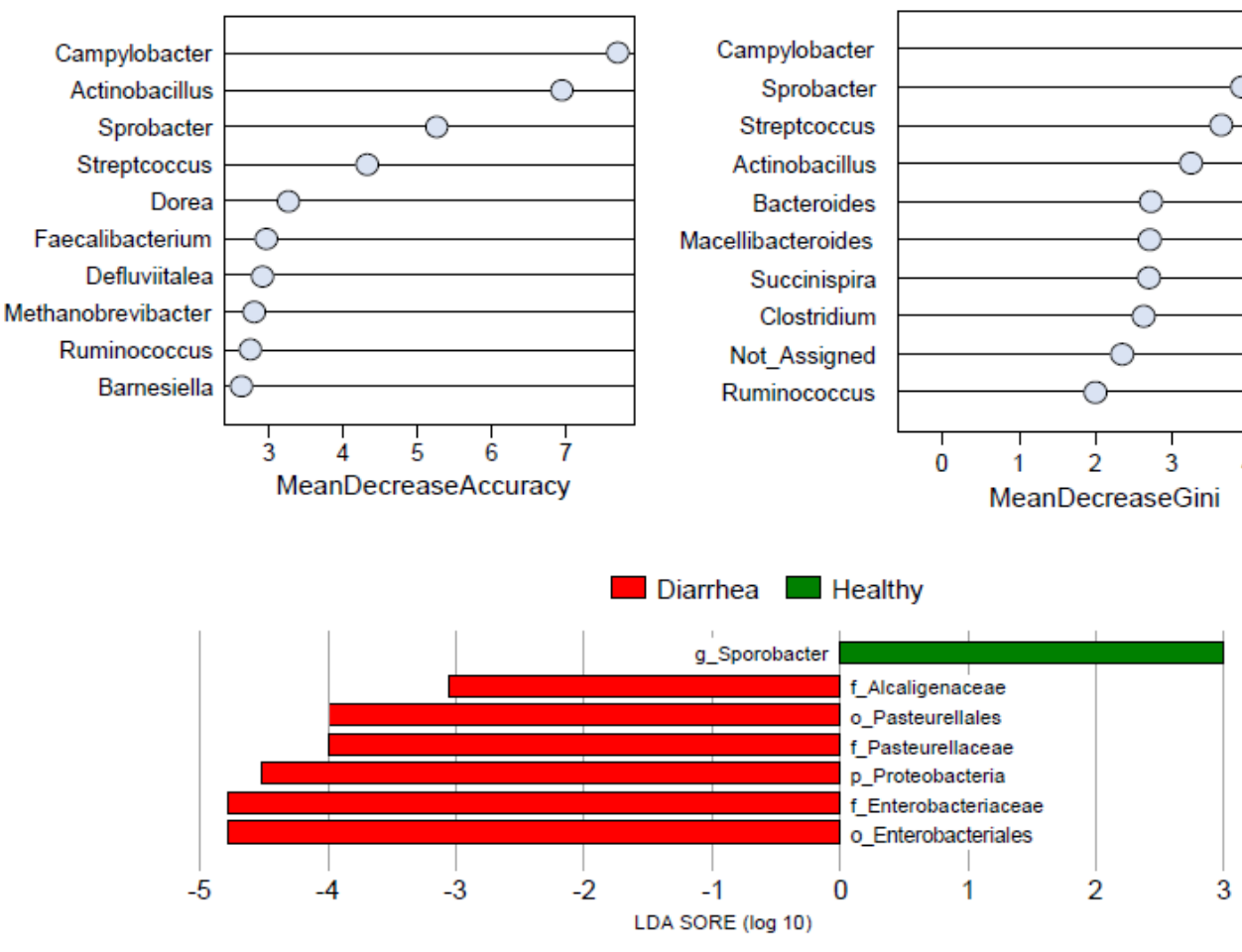

Figure 7

Rational donor selection for successful FMT prediction. (A) PCoA based on unweighted UniFrac distance of overall healthy and diarrheal calves used in FMT. Random-forest classification based on (B) mean decrease accuracy and (C) mean decrease gini. (D) Linear discriminant analysis (LDA) score obtained 
from linear discriminant analysis shown for healthy $(n=105)$, diarrheal $(n=46)$, D-success $(n=13)$ and R-0-success $(n=13)$ from successful FMT, D-failure $(n=4)$ and R-0-failure $(n=13)$ from unsuccessful FMT trial.

\section{Supplementary Files}

This is a list of supplementary files associated with this preprint. Click to download.

- AdditionalFile1.pdf

- AdditionalFile2.pdf

- AdditionalFile3.xlsx 